\title{
Review on Design of HDLC Protocol using HDL
}

\author{
Ku. Rupal P. Bende ${ }^{1}$, Prof. A. P. Bagade ${ }^{2}$, Prof. S. R. Salwe ${ }^{3}$ \\ M.Tech Student, Department of Electronics and Telecommunication, B.D.C.O.E, Wardha, India ${ }^{1}$ \\ Assistant Professor, Department of Electronics and Telecommunication, B.D.C.O.E, Wardha, India ${ }^{2,3}$
}

\begin{abstract}
Protocols are needed for efficient data communication over network. They are specified for each layer of the OSI or TCP/IP reference model by standards engineering organization. HDLC i.e. High level data link control protocol is one of the protocols defined by International Organization for Standardization (ISO) for data link layer of OSI reference model. HDLC is bit oriented protocol and it is most widely used in the network. There are different methods of designing and implementing the protocol. This paper presents different approaches of designing the HDLC protocol using hardware description language and its implementation using software tools.
\end{abstract}

Keywords: HDLC, VHDL, FCS, FPGA, Verilog HDL, Xilinx.

\section{INTRODUCTION}

HDLC stands for High level data link control protocol. It Thus this paper describes the review work on design of is a bit orientated protocol and sends information as a HDLC protocol using hardware description language and sequence of bits. HDLC protocol is specified for data link its implementation by using the different software tools.

layer of OSI reference model. HDLC protocol is a common protocol in networking. SDLC protocol was adopted by IEEE and defined as HDLC. This protocol is used to send data over communication channel.

It was developed by the International Organization for Standardization (ISO). It falls under the ISO standards ISO 3309 and ISO 4335. It sends the data in the form of frames. The frame has to follow a predefined format called as frame structure. Every frame starts and ends with flag sequence field. Then address field holds the address of the receiving station. Control field holds the control code. Data field is the information to be transferred. Frame Check Sequence FCS adopts the CRC as calculating polynomial. The control field varies depending on the mode of operation. A frame contains error checking information which allows data to be sent reliably from a sender to a receiver

There are three modes of operations: Normal Response Mode (NRM), Asynchronous Response Mode (ARM), Asynchronous Balanced Mode (ABM). HDLC specifies the following three types of stations for data link control: 1.Primary Station, Secondary Station, Combined Station. HDLC also defines three types' ofconfigurations for the three types of stations: Unbalanced Configuration Balanced Configuration, Symmetrical Configuration.

It specifies a packetization standard for serial links. It has found itself being used throughout the world. It has been so widely implemented because it supports both halfduplex and full-duplex communication lines, point-topoint and multi-point networks, and switched or nonswitched channels. Other benefits of HDLC are that the control information is always in the same position, and specific bit patterns used for control differ from those in representing data, which reduces the chance of errors. If errorsoccurs, it is detected at the receiver side. It has also led to many subsets. Two subsets widely in use are Synchronous Data Link Control (SDLC) and Link Access Procedure-Balanced (LAP-B).

\section{LITERATURE REVIEW}

The research papers on the design of HDLC protocols are published in various journals and presented in many conferences. Here the paper selected describes the design of protocol using VHDL or Verilog language which includes the design of transmitter and receiver. Some of the papers present the design of protocol for particular application. They have designed the protocol according to the requirements of application. The clock requirement; synchronization requirement for every design is different. The CRC polynomial selected can be 16-bit or 32bit.Mostly CRC-16 has been used.Most papers have used FSMs to design the transmitter and receiver of their controller.

Syed Manzoor Qasim and Shuja A. Abbasi [1] presented the design of single channel HDLC protocol. They designed the transmitter, coded in VHDL and implemented in Xilinx Virtex FPGA. The HDLC Transmitter consists of the following main blocks as shown in figure 1:-Transmitter Controller Transmit Register, Address Insertion, Frame Check Sequence (FCS) generation, Bit Stuffer, Flag/Abort Generation.

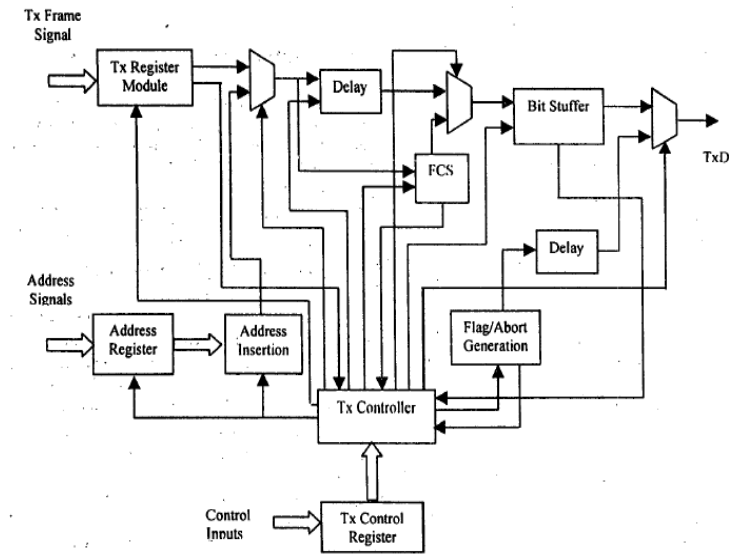

Fig.1 Block diagram of transmitter 
They achieved frequency of $59.2 \mathrm{MHz}$ with a total transceiver consists of the following main blocks:-Control equivalent gate count 574. It is suitable for Frame Relay unit and Registers, Transmitter, Receiver, Dual port switches, Video conferencing on ISDN, ISDN B-channel, RAMS and RAM management unit, Interrupt Controller. SONET Termination, X.25 layer-2 protocol, Cable Figure 4 shows the block diagram of HDLC transceiver. Modem, Private packet data networks \& switches.

S. Hamed Javadi and Ali Peiravi [2] designed the HDLC transceiver and implemented in Xilinx VirtexII FPGA. The maximum bit rate of $85 \mathrm{MHz}$ achieved. The signals of controller are compatible with MT8952 produced by Zarlink which are used in industry and also compatible with ST bus format. The designed HDLC controller uses the available resources of the target technology efficiently, so it is possible to integrate it with other systems. This HDLC controller is based on a modified MT8952B controller as shown in figure 2.

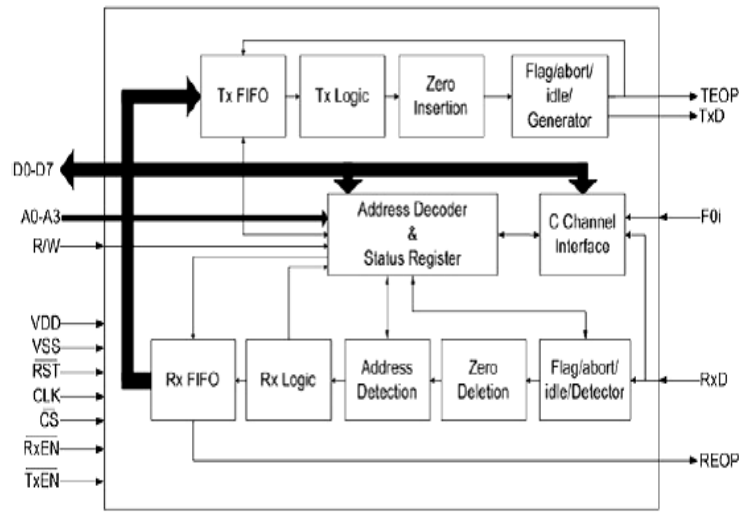

Fig. 2HDLC controller

This transceiver is useful in packet switchingand ISDN for its D-channel. K. Sakthidasan, Mohammed Mahommed [3] presented the design of transceiver in VHDL and implemented in Xilinx virtex FPGA. The codes were simulated by Modelsim. It can be used in MODEMS where error detection part is performed by this HDLC controller. It also support ISDN frame format and thus the flexibility in this controller is very high. The block diagram of controller designed is shown in figure 3 .

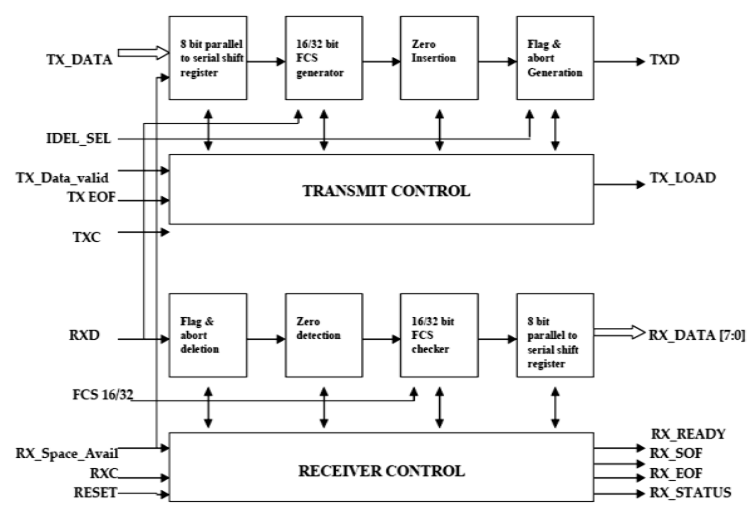

Fig. 3 Block diagram of controller frequency of 70.64 Mbps. The design was coded in Verilog HDL and synthesized with Xilinx Virtex FPGA as target device. Its functional simulation was carried out using Modelsim simulator. The HDLC protocol shown below:-

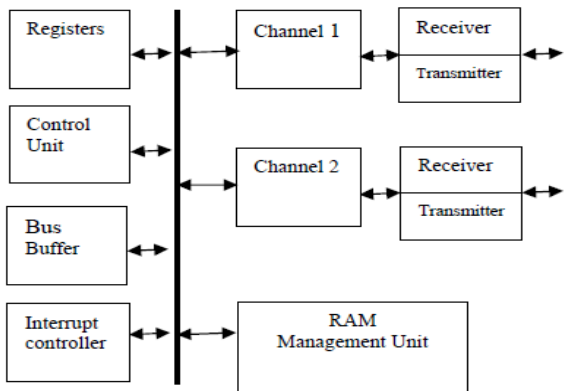

Fig. 4 Multichannel HDLC transceiver

The transceiver is designed for the full duplex communication. The authors of paper [5] presented the design of HDLC transceiver in Verilog HDL language. They implemented the design in VCS synpsys simulation tool.The proposed HDLC transmitter has 3 components, which are- FIFO, zero stuffing and transmission. Figure 5 shows the block diagram of the transmitter.

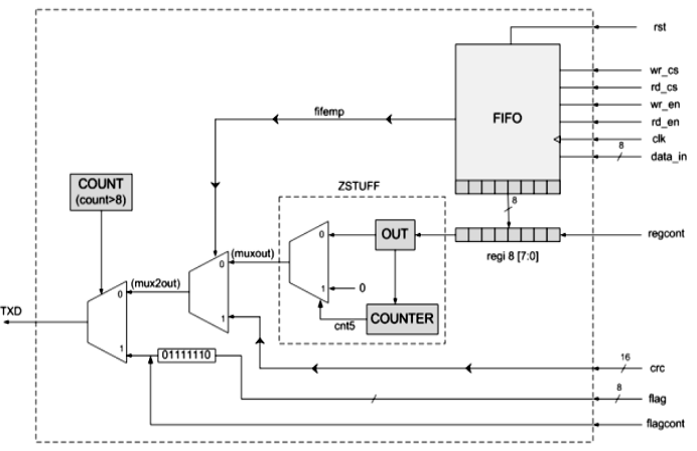

Fig. 5 HDLC transmitter

The architecture for the HDLC receiver is shown in figure 6:-

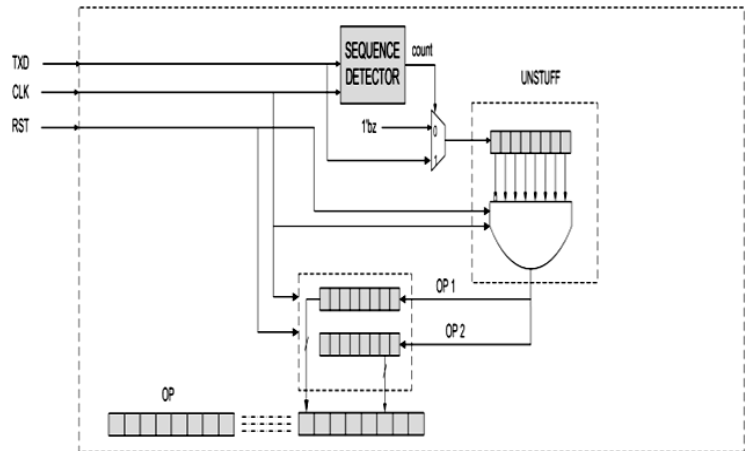

Fig. 6 HDLC receiver

Gaurav Chandil, Priyanka Mishra [6] presented design of HDLC controller. The coding done in VHDL and implemented by using FPGA Spartan 3 device. It offers highest frequency of $155.52 \mathrm{Mbps}$.The signals of thiscontroller are compatible with ITU q.921.X.25 level 2 recommendation. The block diagram of transceiver is 
INTERNATIONAL JOURNAL OF INNOVATIVE RESEARCH IN ELECTRICAL, ELECTRONICS, INSTRUMENTATION AND CONTROL ENGINEERING Vol. 4, Issue 2, February 2016

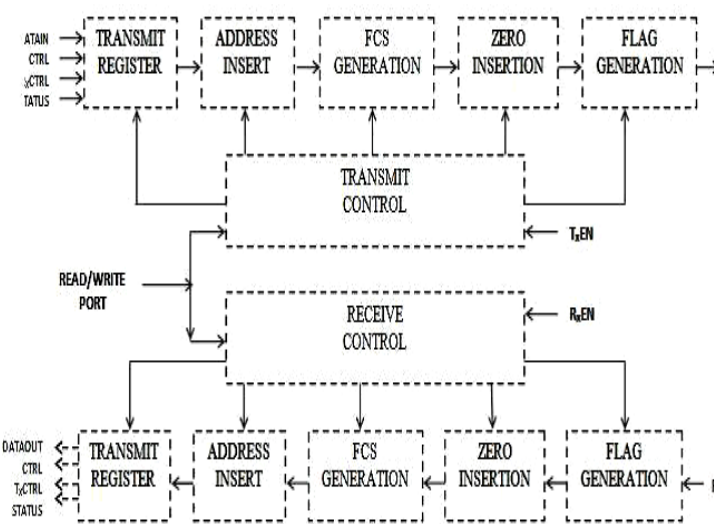

Fig. 7 HDLC transceiver

HDLC controller design supports full Duplex and half duplex mode of operations.It is CCITT X.25 compatible. Jai Karan Singh1, Mukesh Tiwari, Mohd Firoz Warsi proposed a broadband radar system communication [7] in which control computer gives instruction and also receives the results from radar signal processor ,HDLC controller exchange the data between radar signal processor and control computer. Figure 8 shows the block diagram of the system. HDLC controller is designed according to communication requirements of baud rate less than 4 $\mathrm{Mb} / \mathrm{s}$, the communication distance does not exceed 10 meters, the error rate less than 10-6. The designed HDLC controller has the following features: receiving and sending module programming control, query/interrupt working mode, 8-bit station address freely set, data overflow/CRC error detection, DDR-SRAM memory.

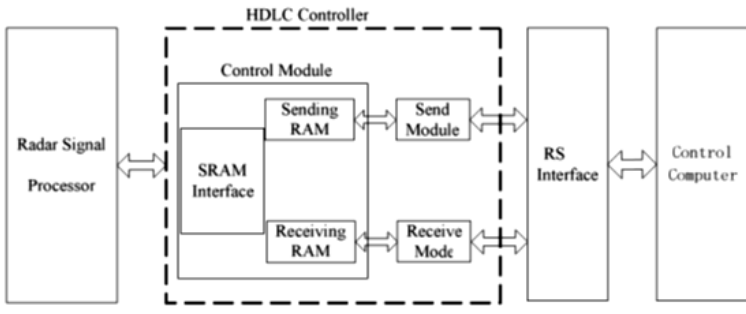

Fig. 8 Block diagram of Radar communication system
HDLC controller designed in Verilog with ISE 12.1 and simulated in Modelsim 6.3. Shubham Fadnavis [8] designed HDLC protocol for error detection and correction. This is application based paper which presents high efficient combined error detection and correction technique based on horizontal-vertical diagonal parity check in HDLC. This method has been experimentally implemented and simulated using field programmable gate array. Simulation results show that the proposed technique detects $99.99 \%$ of the errors and corrects as predicted up to three bits of errors in the received impaired n-bit code. The design was implemented in Xilinx 8.1 using Modelsim SE-EE for simulation.

The authors [9] designed the HDLC protocol for data communication. In designed communication system, data is first compressed, transmitted by using HDLC frame and received by the receiver. To select the 32 Channels information, multiplexer is used. The system has 32 bit multiplexer, channel counter, FIFO (first in first out), RLE Compressor and HDLC Framing at the transmission end. A 32 bit de-multiplexer, FIFO (first in first out), RLE Decompressor and a HDLC De-framing at the receiving end. The architecture of system is shown in figure 9.

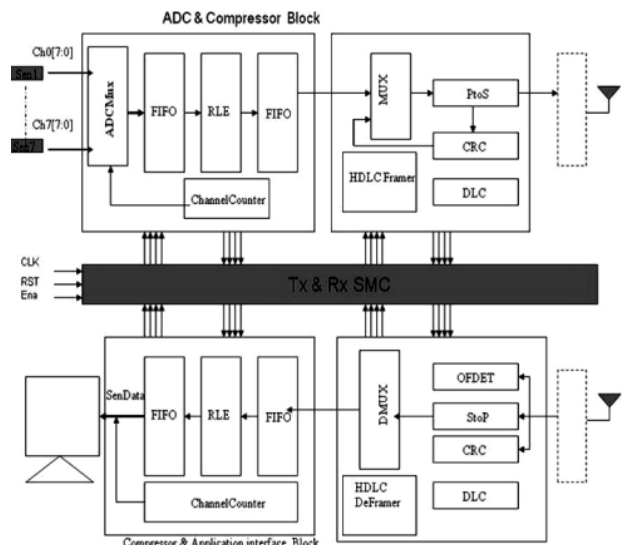

Fig. 9 Block diagram

All the modules are coded in VHDL and implemented in Xilinx Spartan-3 device.

\section{COMPARISON TABLE}

\begin{tabular}{|c|c|l|l|}
\hline Ref. paper No & Bit rate & \multicolumn{1}{|c|}{ Features } & \multicolumn{1}{|c|}{ Software tool used } \\
\hline$[1]$ & $59.2 \mathrm{Mbps}$ & 1.Transmitter is designed & Xilinx Virtex FPGA, Modelsim \\
\hline$[2]$ & $85 \mathrm{Mbps}$ & $\begin{array}{l}\text { Compatible with ST bus format, MT8952 } \\
\text { ZARLINK HDLC Controller, Transceiver } \\
\text { designed. }\end{array}$ & Xilinx VirtexII FPGA, Modelsim \\
\hline$[3]$ & - & Transceiver designed & Xilinx Virtex FPGA, Modelsim \\
\hline$[4]$ & $70.64 \mathrm{Mbps}$ & Multichannel transceiver is designed & Xilinx Virtex FPGA, Modelsim \\
\hline$[5]$ & - & Transceiver designed by Verilog hdl. & VCS Synopsys \\
\hline$[6]$ & 155.52 Mbps & Transceiver designed. & FPGA Spartan 3 device., Modelsim \\
\hline$[7]$ & $4 \mathrm{Mbps}$ & $\begin{array}{l}\text { 1. HDLC controller is designed for radar } \\
\text { communication }\end{array}$ & XILINX ISE 12.1, Modelsim 6.3. \\
\hline$[8]$ & - & $\begin{array}{l}\text { 1. This technique detects 99.99\% of the errors } \\
\text { 2. It corrects as predicted up to three bits of } \\
\text { errors }\end{array}$ & Xilinx 8.1, Modelsim SE-EE 5.4A \\
\hline$[9]$ & - & $\begin{array}{l}\text { 1. They designed the system in which data is } \\
\text { compressed then transmitted and received } \\
\text { using HDLC }\end{array}$ & Xilinx Spartan-3 \\
\hline & &
\end{tabular}




\section{CONCLUSION}

The study of papers shows different approaches of designing the HDLC protocol using hardware description language. It has been found that HDLC controller for transmitter and receiver is designed in VHDL or Verilog language and then implemented using various software tools. The authors designed protocol at the different bit rate and highest bit rate of $155.52 \mathrm{Mbps}$ is achieved. It is also observed that the HDLC protocol is designed according to the requirements of particular application. Thus this review gives brief idea that by designing the HDLC protocol in hardware description language, the protocol can be made more efficient and its speed can be increased. Hence it can be used in more application, making it more flexible and upgradable.

\section{ACKNOWLEDGEMENT}

We would like to thanks Mr. P.R. Indurkar, Associate Professor, Electronics and Telecommunication department, B.D.C.O.E for his valuable suggestions. We would thanks to our college for providing valuable facilities which helps us in our research work. We also express thanks to our parents, friends and colleagues.

\section{REFERENCES}

[1] Syed Manzoor Qasim and Shuja A. Abbasi ," FPGA Implementation of a Single-Channel HDLCLayer-2 Protocol Transmitter using VHDL", International Conference on Electrical, Electronics and System Engineering, 2003.

[2] S. Hamed Javadi and Ali Peiravi,'Design and Implementation of a High Bit Rate HDLCTransceiver Based on a Modified MT8952B Controller", Australian Journal of Basic and Applied Sciences, 2009

[3] K. Sakthidasan, Mohammed Mahommed,"Design of HDLC Controller Using VHDL", International Journal of Scientific \& Engineering Research Volume 2, Issue 3, March-2011.

[4] Harpreet Singh, Navneet Kaur, Vinay Chopra and Dr.Amardeep Singh, "Optimization of multi - channel HDLC protocol transceiver using Verilog", International Journal of Computer Science Issues, Vol. 9, Issue 2, No 2, March 2012.

[5] Armaan Hasan Nagpurwala, Sundaresan C, Chaitanya CVS, "Implementation of HDLC Controller Design using Verilog HDL “, International Conference on Electrical, Electronics and System Engineering,2013.

[6] Gaurav Chandil, Priyanka Mishra,'Design and Implementation of HDLC Controller by Using Crc-16", International Journal of Modern Engineering Research (IJMER) Jan.-Feb. 2013.

[7] Jai Karan Singh1, Mukesh Tiwari, Mohd Firoz Warsi," Implementation of HDLC Protocol Based DDR-RAM Radar Processing System", International Journal of VLSI and Embedded Systems-IJVES -May - June 2013..

[8] Shubham Fadnavis,"An HVD Based Error Detection and Correction Code in HDLC Protocol Used for Communication", International Journal of Advanced Research in Computer and Communication Engineering, Vol. 2, Issue 6, June 2013.

[9] Shashank Rampelly, Santhosh Rao Seri, Gnaneshwara Chary, Krishanm Raju, "Data Communication Using HDLC Protocol", International Journal of Innovative Research in Electrical, Electronics, Instrumentation and control engineering, Vol. 2, Issue 5, May 2014.

[10] Gao, Zhen-bin and Jian-FeiLiu, "FPGA implementation of a multichannel HDLC protocol transceiver", In Proceedings of the 2005 International Conference on Communications, Circuits and Systems, 2: 1300-1302,2005.

[11] Lu, Y., Z. Wang, L. Qiao and B. Huanq, "Design and implementation of multi-channel high speed HDLC data processor," IEEE International Conference on Communications,
Circuits and Systems, and West Sino Expositions, 2: 14711475,2002.

[12] Jun Wang; Wenhao Zhang; Yuxi Zhang; Wei Wu; Weiguang Chang; Sch. of Electron. \& Inf. Eng., Beihang Univ. (BUAA), Beijing, China "Design and implementation of HDLC procedure based on FPGA", Anti- ounterfeiting, Security, and Identification in Communication, ASID, 3rd International Conference, 20-22 Aug.2009.

[13] Wang Lie, Yi Mingvol, "Design of HDLC Controller based on XILINX FPGA“ International Conference on Electrical, Electronics and System Engineering IEEE, 2011. 